\title{
Colpitts Chaotic Oscillator Coupling with a Generalized Memristor
}

\author{
Ling Lu, Changdi Li, Zicheng Zhao, Bocheng Bao, and Quan Xu \\ School of Information Science and Engineering, Changzhou University, Changzhou 213164, China
}

Correspondence should be addressed to Bocheng Bao; mervinbao@126.com

Received 1 October 2014; Revised 24 February 2015; Accepted 16 March 2015

Academic Editor: Franck J. Vernerey

Copyright (C) 2015 Ling Lu et al. This is an open access article distributed under the Creative Commons Attribution License, which permits unrestricted use, distribution, and reproduction in any medium, provided the original work is properly cited.

By introducing a generalized memristor into a fourth-order Colpitts chaotic oscillator, a new memristive Colpitts chaotic oscillator is proposed in this paper. The generalized memristor is equivalent to a diode bridge cascaded with a first-order parallel RC filter. Chaotic attractors of the oscillator are numerically revealed from the mathematical model and experimentally captured from the physical circuit. The dynamics of the memristive Colpitts chaotic oscillator is investigated both theoretically and numerically, from which it can be found that the oscillator has a unique equilibrium point and displays complex nonlinear phenomena.

\section{Introduction}

Memristor, regarded as a fourth circuit element, is a passive two-terminal electronic element characterized by nonlinear constitutive relation of charge and flux $[1,2]$. Due to the nonlinearity, memristor-based application circuits can easily generate chaotic signals, which immensely inspire research interests in the designs of various memristive chaotic circuits. In recent years, memristor-based chaotic circuits, most of all memristive Chua's chaotic circuits, have broadly been studied [3-10]. By substituting the nonlinear resistance elements, Chua's diodes, in various kinds of Chua's chaotic circuits, including classical Chua's circuit [3-8], canonical Chua's circuit [9-11], and modified Chua's circuit [12, 13], with memristors of different nonlinearities $[4,7,12]$, a wide class of memristive Chua's chaotic circuits are presented and some novel features of initial state dependent dynamical behaviors are reported $[3,5,10,11]$. However, no research literature related to memristor-based Colpitts chaotic oscillator can be found so far.

The third-order or fourth-order Colpitts oscillator is a single-transistor implementation of a sinusoidal oscillation circuit widely used in electronic circuits and communication systems $[14,15]$. The operation frequency can vary from a few hertz up to the microwave region (gigahertz), depending on the making technology. The system possesses an intrinsic nonlinearity given by the exponential [14] or piece-wise linear [15] characteristic of the active device. The Colpitts oscillator can exhibit rich dynamical behaviors like many other oscillator configurations analyzed in the literatures. In particular, for the Colpitts oscillator there are extensive numerical and experimental evidences of nonlinear behaviors $[14,15]$. Therefore, by introducing a memristor into the fourth-order Colpitts oscillator [15], a novel memristive Colpitts oscillator maintaining with the original advantages can easily be constructed.

An analogue memristor model can be realized by using a light-dependent resistor (LDR) circuit $[16,17]$. In the same way, a simple analogue memristor model can be implemented by utilizing a diode bridge circuit $[18,19]$. In this paper, a first-order generalized memristor consisting of a diode bridge cascaded with a first-order parallel RC filter [18] is utilized to implement the proposed memristive Colpitts oscillator, which is simplified from a second-order generalized memristor realized by a memristive diode bridge with LCR filter [19]. The first-order generalized memristor is composed of only elementary electronic circuit elements and is well suitable for experimental measurements. Based on the proposed memristive Colpitts chaotic oscillator, dynamical modeling, numerical simulations, and experimental observations are performed in order. 


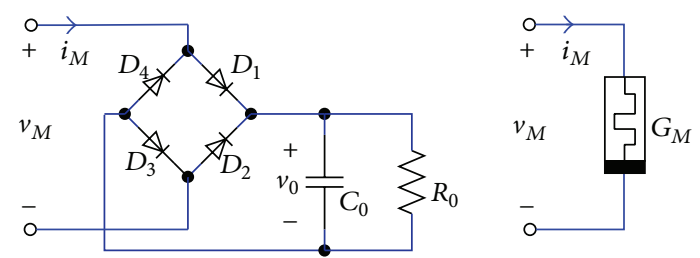

FIGURE 1: Generalized memristor realized by a diode bridge with parallel RC filter.

\section{Memristive Colpitts Chaotic Oscillator}

2.1. Generalized Memristor. The equivalent realization circuit of the first-order generalized memristor in [18] is shown in Figure 1, which is realized by a diode bridge cascaded with a first-order parallel RC filter. The key mechanisms at the origin of its memristive behavior are the voltage constraints involving each pair of parallel diodes [19].

Assuming that $v_{M}$ and $i_{M}$ represent the voltage across and the current flowing through the input terminal of the generalized memristor and $v_{0}$ is the voltage of the capacitor $\mathrm{C}_{0}$, the mathematical model can be expressed as

$$
\begin{gathered}
i_{M}=G_{M} v_{M}=2 I_{S 1} e^{-\rho_{1} v_{0}} \sinh \left(\rho_{1} v_{M}\right), \\
\frac{\mathrm{d} v_{0}}{\mathrm{~d} t}=\frac{2 I_{S 1} e^{-\rho_{1} v_{0}} \cosh \left(\rho_{1} v_{M}\right)}{C_{0}}-\frac{v_{0}}{R_{0} C_{0}}-\frac{2 I_{S 1}}{C_{0}},
\end{gathered}
$$

where $\rho_{1}=1 /\left(2 n_{1} V_{T}\right)$ and $I_{S 1}, n_{1}$, and $V_{T}$ are the reverse saturation current, emission coefficient, and thermal voltage of the diode, respectively. In this paper, four 1 N4148 diodes are utilized in Figure 1 and the diode parameters are determined by $I_{S 1}=5.84 \mathrm{nA}, n_{1}=1.94$, and $V_{T}=25 \mathrm{mV}$.

The mathematical model described by (1) and (2) accords with the defining equations for the class of generalized memristors [20]. The generalized memristor is indeed voltagecontrolled and its memductance is expressed as

$$
G_{M}=\frac{2 I_{S 1} e^{-\rho_{1} v_{0}} \sinh \left(\rho_{1} v_{M}\right)}{v_{M}} .
$$

Consider that the circuit parameters in Figure 1 are $R_{0}=$ $200 \Omega$ and $C_{0}=2 \mu \mathrm{F}$ and the applied voltage is a bipolar sinusoidal voltage of $v_{M}=V_{m} \sin (2 \pi f t) \mathrm{V}$. When $V_{m}=$ $2 \mathrm{~V}$ and $f$ is set to $1 \mathrm{kHz}$ and to $5 \mathrm{kHz}$, respectively, the generalized memristor can display hysteresis loops pinched at the origin, as shown in Figure 2(a). It can be seen that the area of the pinched hysteresis lobe decreases monotonically as the frequency of the periodic input voltage increases and the pinched hysteresis loop shrinks to a nonlinear single-valued function when the frequency tends to infinity. Therefore, the generalized memristor can exhibit three characteristic fingerprints for identifying memristors [21]. When $f=1 \mathrm{kHz}$ and two voltages of $v_{M}=2 \sin (2 \pi f t)+1.2 \cos (5 \pi f t) \mathrm{V}$ and $v_{M}=2.5+1.5 \sin (2 \pi f t) \mathrm{V}$ are applied, the loci in the $v_{M}-i_{M}$ plane are shown in Figure 2(b), where, for the applied voltage with the two different frequencies, the locus is a double-loop hysteresis pinched at the origin; however, for the applied voltage with unipolarity, the locus is a single-loop hysteresis and is not pinched at the origin. Thus, for different voltage stimuli, the loci of the generalized memristor are the hysteresis loops with different shapes.

For the generalized memristor shown in Figure 1, it is remarkable that

(1) the pinched hysteresis loop is independent of the initial condition of the inner state variable $v_{0}$,

(2) the effective operating frequency range depends on the inner circuit parameters, that is, the time constant $R_{0} C_{0}$, and will be higher for the smaller time constant,

(3) the smaller resistance $R_{0}$ can generate the bigger current $i_{M}$ flowing through the generalized memristor.

2.2. Memristive Colpitts Chaotic Oscillator. It was reported in [15] that a fourth-order Colpitts oscillator can generate chaos. When a generalized memristor is introduced into the Colpitts oscillator, a novel memristor-based chaotic circuit is easily designed, as shown in Figure 3, in which the generalized memristor represents the memristive diode bridge circuit shown in Figure 1 . Considering the inner state variable $v_{0}$ in the generalized memristor, the memristive Colpitts oscillator is a fifth-order circuit.

An NPN bipolar junction transistor is included as a nonlinear element in the Colpitts oscillator. When parasitic dynamics of the transistor are neglected, an intrinsic nonlinearity given by the exponential characteristic is considered to model for the active device and the common-base forward short-circuit current gain equals 1 . Two parameters $I_{S 2}$ and $V_{T}$ are used for the descriptions of the transport saturation current and thermal voltage of the transistor Q, respectively. For the transistor $\mathrm{Q}$, the mathematical model is featured by [14]

$$
i_{e}=I_{S 2}\left(e^{v_{\mathrm{be}} / V_{T}}-1\right)=I_{S 2}\left(e^{\rho_{2} v_{1}}-1\right),
$$

where $\rho_{2}=1 / V_{T}$ and $i_{e}$ is the emitter current of the transistor. When the type of $2 \mathrm{~N} 2222$ is considered in Figure 2, the transistor parameters are determined by $I_{S 2}=3.0484 \mathrm{nA}$ and $V_{T}=25 \mathrm{mV}$.

There are four state variables of the capacitor $C_{1}$ voltage $v_{1}$, the capacitor $C_{2}$ voltage $v_{2}$, the capacitor $C_{3}$ voltage $v_{3}$, and the inductor $L$ current $i_{L}$ in the fourth-order Colpitts oscillator and one state variable of the capacitor $C_{0}$ voltage $v_{0}$ in the generalized memristor. Applying Kirchhoff's circuit laws and the constitutive relations of circuit elements, a state equation set can be obtained as

$$
\begin{gathered}
C_{1} \frac{\mathrm{d} v_{1}}{\mathrm{~d} t}=i_{M}-i_{R}-\frac{V_{E E}+v_{1}}{R_{2}}, \\
C_{2} \frac{\mathrm{d} v_{2}}{\mathrm{~d} t}=i_{R}-i_{M}-i_{e}, \\
C_{3} \frac{\mathrm{d} v_{3}}{\mathrm{~d} t}=i_{R}-i_{M}-i_{L}, \\
L \frac{\mathrm{d} i_{L}}{\mathrm{~d} t}=v_{3}, \\
C_{0} \frac{\mathrm{d} v_{0}}{\mathrm{~d} t}=2 I_{S 1} e^{-\rho_{1} v_{0}} \cosh \left(\rho_{1} v_{M}\right)-2 I_{S 1}-\frac{v_{0}}{R_{0}}
\end{gathered}
$$




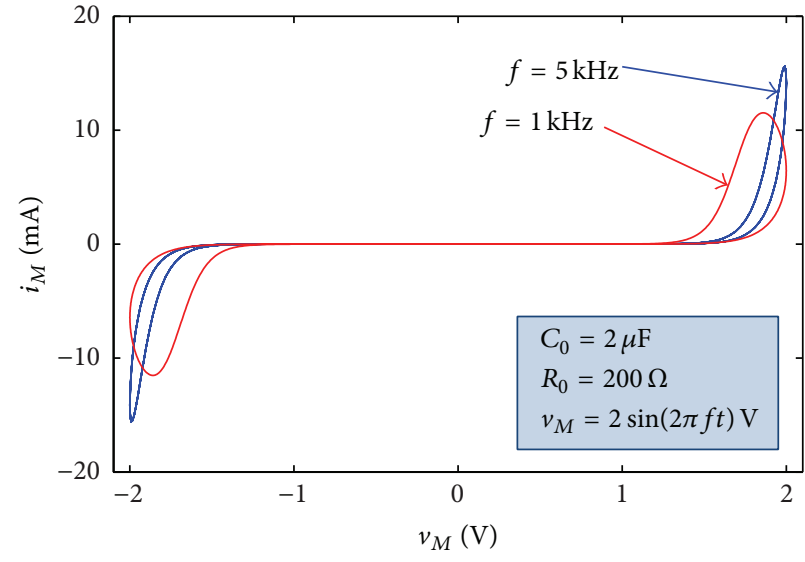

(a)

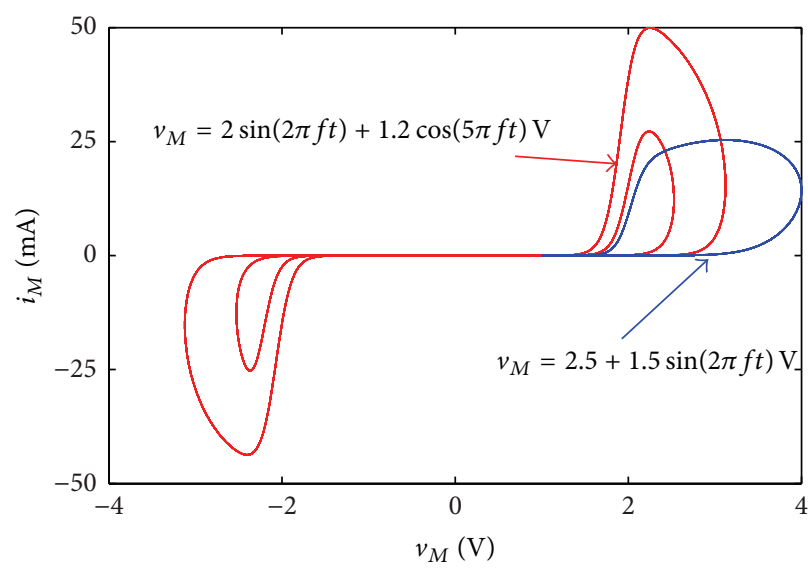

(b)

Figure 2: Loci in the $v_{M}-i_{M}$ plane of the generalized memristor driven by different input voltages: (a) bipolar sinusoidal voltage stimuli; (b) other periodic voltage stimuli.

where $i_{b}=0$ and $i_{c}=i_{e}$ are considered, and

$$
\begin{gathered}
v_{M}=-v_{1}+v_{2}+v_{3}, \\
i_{M}=2 I_{S 1} e^{-\rho_{1} v_{0}} \sinh \left(\rho_{1} v_{M}\right), \\
i_{R}=\frac{\left(V_{C C}-v_{M}\right)}{R_{1}} .
\end{gathered}
$$

Substituting (4) and (6) into (5) yields

$$
\begin{gathered}
\frac{\mathrm{d} v_{1}}{\mathrm{~d} t}=\frac{2 I_{S 1} e^{-\rho_{1} v_{0}} \sinh \left(\rho_{1} v_{M}\right)}{C_{1}}-\frac{V_{C C}-v_{M}}{R_{1} C_{1}}-\frac{V_{E E}+v_{1}}{R_{2} C_{1}}, \\
\frac{\mathrm{d} v_{2}}{\mathrm{~d} t}=\frac{V_{C C}-v_{M}}{R_{1} C_{2}}-\frac{2 I_{S 1} e^{-\rho_{1} v_{0}} \sinh \left(\rho_{1} v_{M}\right)}{C_{2}}-\frac{I_{S 2}\left(e^{\rho_{2} v_{1}}-1\right)}{C_{2}}, \\
\frac{\mathrm{d} v_{3}}{\mathrm{~d} t}=\frac{V_{C C}-v_{M}}{R_{1} C_{3}}-\frac{2 I_{S 1} e^{-\rho_{1} v_{0}} \sinh \left(\rho_{1} v_{M}\right)}{C_{3}}-\frac{i_{L}}{C_{3}}, \\
\frac{\mathrm{d} i_{L}}{\mathrm{~d} t}=\frac{v_{3}}{L}, \\
\frac{\mathrm{d} v_{0}}{L}=\frac{2 I_{S 1} e^{-\rho_{1} v_{0}} \cosh \left(\rho_{1} v_{M}\right)}{C_{0}}-\frac{v_{0}}{R_{0} C_{0}}-\frac{2 I_{S 1}}{C_{0}} .
\end{gathered}
$$

Equation (7) is a five-dimensional nonlinear system, upon which dynamical analysis of the memristive Colpitts oscillator can be performed numerically.

\section{Dissipativity and Stability Analysis}

3.1. Dissipativity. The memristive Colpitts oscillator in Figure 3 is dissipative. The dissipativity can be derived from

$$
\begin{aligned}
\nabla V= & \frac{\partial \dot{v}_{1}}{\partial v_{1}}+\frac{\partial \dot{v}_{2}}{\partial v_{2}}+\frac{\partial \dot{v}_{3}}{\partial v_{3}}+\frac{\partial \dot{i}_{L}}{\partial i_{L}}+\frac{\partial \dot{v}_{0}}{\partial v_{0}} \\
= & -2 \rho_{1} I_{S 1} e^{-\rho_{1} v_{0}} \cosh \left(\rho_{1} v_{M}\right)\left(\frac{1}{C_{1}}+\frac{1}{C_{2}}+\frac{1}{C_{3}}+\frac{1}{C_{0}}\right) \\
& -\frac{1}{R_{1}}\left(\frac{1}{C_{1}}+\frac{1}{C_{2}}+\frac{1}{C_{3}}\right)-\frac{1}{R_{2} C_{1}}-\frac{1}{R_{0} C_{0}} .
\end{aligned}
$$

Considering that the hyperbolic cosine function is positive, (8) can be simplified as

$$
\nabla V<-\frac{1}{R_{1}}\left(\frac{1}{C_{1}}+\frac{1}{C_{2}}+\frac{1}{C_{3}}\right)-\frac{1}{R_{2} C_{1}}-\frac{1}{R_{0} C_{0}}<0 .
$$

Therefore, the dissipativity is always negative, implying that all orbits are ultimately confined to a specific subset of zero volume, and the asymptotic motion settles onto an attractor.

3.2. Equilibrium Point and Its Stability. The equilibrium point of (7) is yielded by solving the following equations:

$$
\begin{gathered}
R_{1} R_{2} i_{M}-R_{2}\left(V_{C C}-v_{M}\right)-R_{1}\left(V_{E E}+v_{1}\right)=0, \\
V_{C C}-v_{M}-R_{1} i_{M}-R_{1} I_{S 2}\left(e^{\rho_{2} v_{1}}-1\right)=0, \\
V_{C C}-v_{M}-R_{1} i_{M}-R_{1} i_{L}=0, \\
v_{3}=0 \\
2 R_{0} I_{S 1} e^{-\rho_{1} v_{0}} \cosh \left(\rho_{1} v_{M}\right)-v_{0}-2 R_{0} I_{S 1}=0 .
\end{gathered}
$$

From (10b) and (10e), we obtain

$$
\begin{aligned}
v_{1} & =\frac{1}{\rho_{2}} \ln \left(\frac{V_{C C}-v_{M}-R_{1} i_{M}}{R_{1} I_{S 2}}+1\right), \\
v_{M} & =\frac{1}{\rho_{1}} \operatorname{arccosh}\left[\left(\frac{v_{0}}{2 R_{0} I_{S 1}}+1\right) e^{\rho_{1} v_{0}}\right] .
\end{aligned}
$$




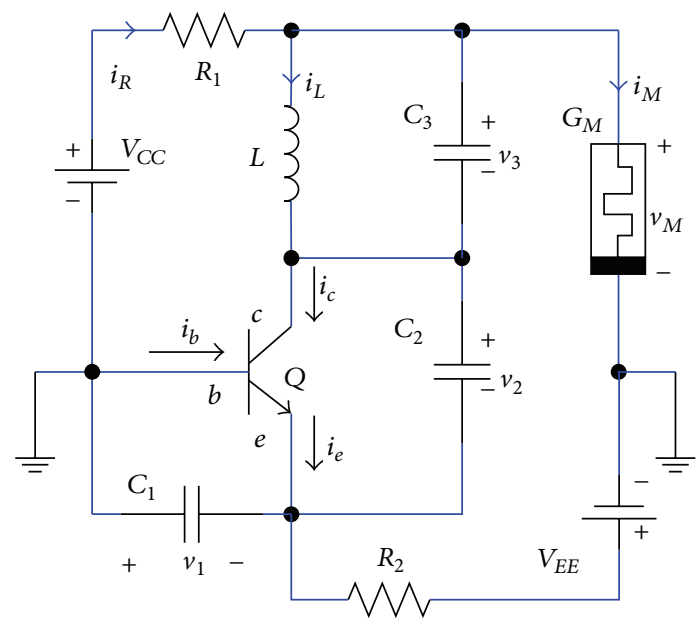

Figure 3: Memristive Colpitts chaotic oscillator based on the generalized memristor.

According to (10a), let

$$
\begin{gathered}
h_{1}=R_{1} R_{2} i_{M}-R_{2}\left(V_{C C}-v_{M}\right), \\
h_{2}=R_{1}\left(V_{E E}+v_{1}\right) \\
=R_{1} V_{E E}+\frac{R_{1}}{\rho_{2}} \ln \left(\frac{V_{C C}-v_{M}-R_{1} i_{M}}{R_{1} I_{S 2}}+1\right) .
\end{gathered}
$$

Substituting (11a) and (11b) into (12a) and (12b) and considering the voltage-current relationship of (1), it can be found that $h_{1}$ and $h_{2}$ are two functions related to $v_{0}$. Letting $h_{1}=$ $h_{2}$, the intersection point of two function curves described by (12a) and (12b) corresponds to the value of $v_{0}$, which represents the location of the equilibrium point on the $v_{0}$ axis and can be obtained through graphic analytic method. Then, the corresponding values of other variables can easily be calculated. For the circuit parameters listed in Table 1, the unique equilibrium point is expressed as

$$
S=(0.3458,4.7217,0,0.0031,2.9460) .
$$

Observed from (10a)-(10e) it can be known that the value of the equilibrium point is mainly determined by the specified circuit parameters of $R_{0}, R_{1}$, and $R_{2}$. This feature is completely different from the conventional memristive chaotic circuits $[3,5,10]$, whose equilibrium points are an equilibrium point set located on the axis corresponding to the inner state variable of the memristor.

The Jacobian matrix of (7), evaluated at the equilibrium point $\bar{S}=\left(\bar{v}_{1}, \bar{v}_{2}, \bar{v}_{3}, \bar{i}_{L}, \bar{v}_{0}\right)$, is given by

$$
\mathbf{J}=\left[\begin{array}{ccccc}
-\frac{a_{1}}{C_{1}}-\frac{1}{R_{2} C_{1}} & \frac{a_{1}}{C_{1}} & \frac{a_{1}}{C_{1}} & 0 & -\frac{a_{2}}{C_{1}} \\
\frac{a_{1}}{C_{2}}-\frac{a_{3}}{C_{2}} & -\frac{a_{1}}{C_{2}} & -\frac{a_{1}}{C_{2}} & 0 & \frac{a_{2}}{C_{2}} \\
\frac{a_{1}}{C_{3}} & -\frac{a_{1}}{C_{3}} & -\frac{a_{1}}{C_{3}} & -\frac{1}{C_{3}} & \frac{a_{2}}{C_{3}} \\
0 & 0 & \frac{1}{L} & 0 & 0 \\
-\frac{a_{2}}{C_{0}} & \frac{a_{2}}{C_{0}} & \frac{a_{2}}{C_{0}} & 0 & \frac{1}{C_{0}}\left(\frac{1}{R_{1}}-\frac{1}{R_{0}}-a_{1}\right)
\end{array}\right],
$$

TABLE 1: Circuit parameters for simulations and experiments.

\begin{tabular}{lcc}
\hline Parameters & Significations & Values \\
\hline$C_{0}, C_{1}, C_{2}$ & Capacitance & $2 \mu \mathrm{F}$ \\
$C_{3}$ & Capacitance & $33 \mathrm{nF}$ \\
$L$ & Inductance & $10 \mathrm{mH}$ \\
$R_{0}$ & Resistance & $200 \Omega$ \\
$R_{1}$ & Resistance & $35 \Omega$ \\
$R_{2}$ & Resistance & $1.5 \mathrm{k} \Omega$ \\
$V_{C C}$ & Direct voltage & $5 \mathrm{~V}$ \\
$V_{E E}$ & Direct voltage & $-5 \mathrm{~V}$ \\
\hline
\end{tabular}

where

$$
\begin{gathered}
a_{1}=2 \rho_{1} I_{S 1} e^{-\rho_{1} \bar{v}_{0}} \cosh \left[\rho_{1}\left(-\bar{v}_{1}+\bar{v}_{2}+\bar{v}_{3}\right)\right]+\frac{1}{R_{1}}, \\
a_{2}=2 \rho_{1} I_{S 1} e^{-\rho_{1} \bar{v}_{0}} \sinh \left[\rho_{1}\left(-\bar{v}_{1}+\bar{v}_{2}+\bar{v}_{3}\right)\right] \\
a_{3}=\rho_{2} I_{S 2} e^{\rho_{2} \bar{v}_{1}}
\end{gathered}
$$

Thus, the corresponding characteristic equation can be written as

$$
\operatorname{det}(\mathbf{1} \lambda-\mathbf{J})=0 .
$$

For the circuit parameters listed in Table 1, the five eigenvalues at the equilibrium point are calculated as

$$
\begin{gathered}
\lambda_{1,2}=5036 \pm j 14554, \\
\lambda_{3,4}=-13124 \pm j 3264, \\
\lambda_{5}=-5710503 .
\end{gathered}
$$

It is found that the memristive Colpitts oscillator in Figure 3 has two complex conjugate roots with positive real parts, two complex conjugate roots with negative real parts, and one negative real root; the equilibrium point consequently is an unstable saddle-focus, resulting in the generation of the spiral chaotic attractor.

\section{Typical Chaotic Attractor}

In Figures 1 and 3, the circuit element parameters are listed in Table 1; the four 1N4148 diodes and the 2N2222 transistor are utilized.

4.1. Numerical Simulations. When the initial values of five state variables are selected as $v_{1}(0)=0.01 \mathrm{~V}, v_{2}(0)=$ $0.01 \mathrm{~V}, v_{3}(0)=0 \mathrm{~V}, i_{L}(0)=0 \mathrm{~A}$, and $v_{0}(0)=0 \mathrm{~V}$, the memristive Colpitts oscillator in Figure 3 is chaotic and displays a spiral chaotic attractor, as plotted in Figure 4. The five Lyapunov exponents are $\mathrm{LE}_{1}=769.9443, \mathrm{LE}_{2}=$ $-0.147, \mathrm{LE}_{3}=-3291.8, \mathrm{LE}_{4}=-13589$, and $\mathrm{LE}_{5}=-184610$, 


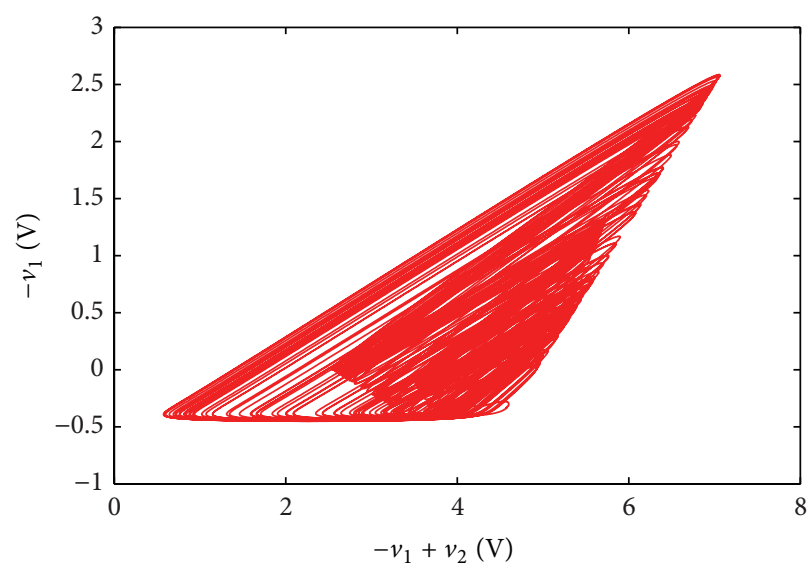

(a)

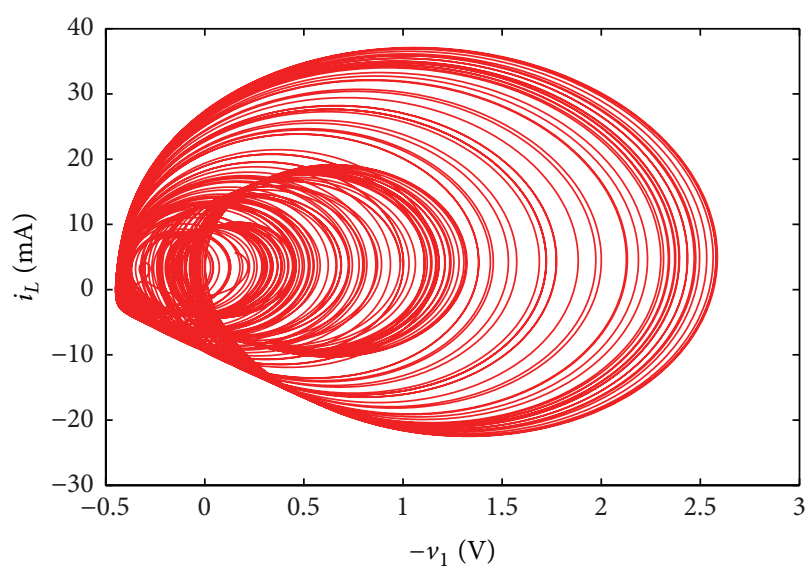

(c)

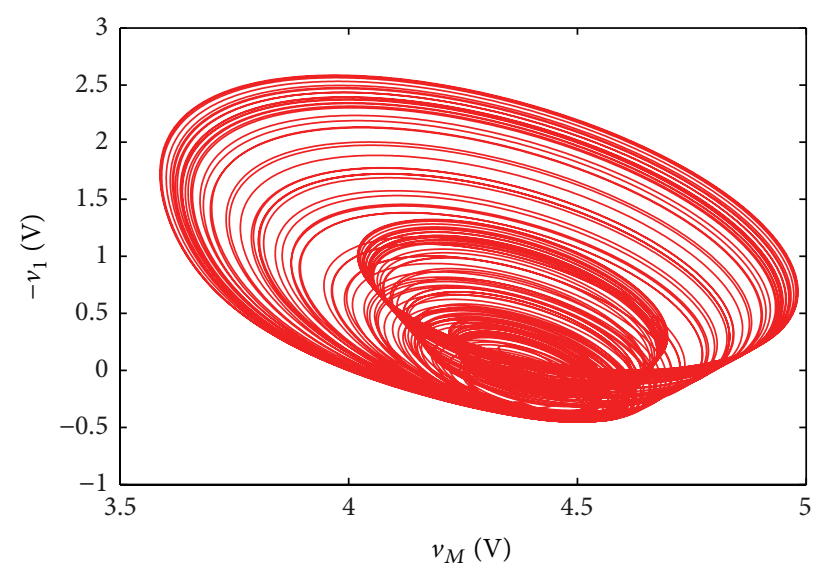

(b)

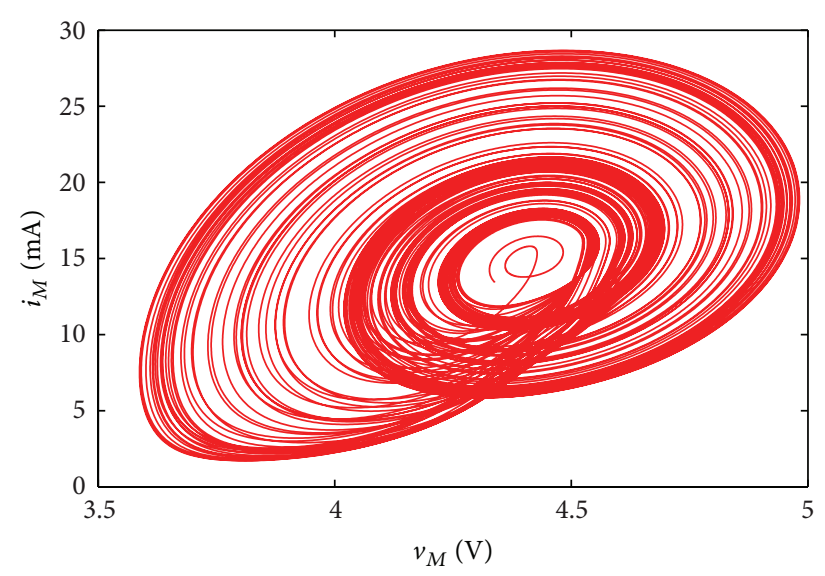

(d)

FIGURE 4: Phase portraits of the memristive Colpitts oscillator by numerical simulations: (a) $-v_{1}+v_{2}$ versus $-v_{1}$; (b) $v_{M}$ versus $-v_{1}$; (c) $-v_{1}$ versus $i_{L} ;(\mathrm{d}) v_{M}$ versus $i_{M}$.

respectively. Note that Figure 4(d) displays the fingerprint of the generalized memristor, which illustrates that there exists a single-loop hysteresis behavior in the generalized memristor due to the applied positive offset voltage with unipolarity.

For the corresponding parameters, the Poincare mappings on $v_{3}(t)=0 \mathrm{~V}$ section are shown in Figures 5(a) and $5(\mathrm{~b})$ and the partial time-domain waveforms of the variables $v_{1}(t), v_{2}(t), i_{M}(t)$, and $v_{M}(t)$ are plotted in Figures $5(\mathrm{c})$ and 5(d). Observed from Figure 5, some folded sheets of the chaotic attractor are visualized and the aperiodic waveforms of the variables are captured. Therefore, the memristive Colpitts oscillator is chaotic, in which complex nonlinear phenomena can easily be observed.

4.2. Experimental Verifications. An experimental setup of the memristive Colpitts oscillator is designed to investigate the dynamical behaviors with the same circuit parameters as for numerical simulations. The chaotic behaviors of the memristive Colpitts oscillator in different phase planes can be experimentally captured in Figure 6. The experimental results in Figure 6 are consistent with the numerical simulations and illustrate that the physical circuit can indeed generate chaos.

\section{Dynamics Depending on Circuit Parameters}

When the resistance $R_{2}$ is a varying parameter, the bifurcation diagram of the state variable $v_{1}(t)$ and the corresponding Lyapunov exponent spectra of the memristive Colpitts chaotic oscillator are depicted in Figures $7(a)$ and 7(b), respectively. For clarity, the first three Lyapunov exponents are presented.

As $R_{2}$ increases gradually within the parameter variation range, the dynamics of the memristive Colpitts chaotic oscillator starts from periodic behaviors and then enters into chaotic behaviors via forward period-doubling bifurcation route and finally settles down periodic behaviors via reverse period-doubling bifurcation route. Correspondingly, the maximum Lyapunov exponent begins with negative region and then reaches positive region and finally returns to negative region. Clearly, several windows of periodicities exist in the band of chaotic behaviors. 


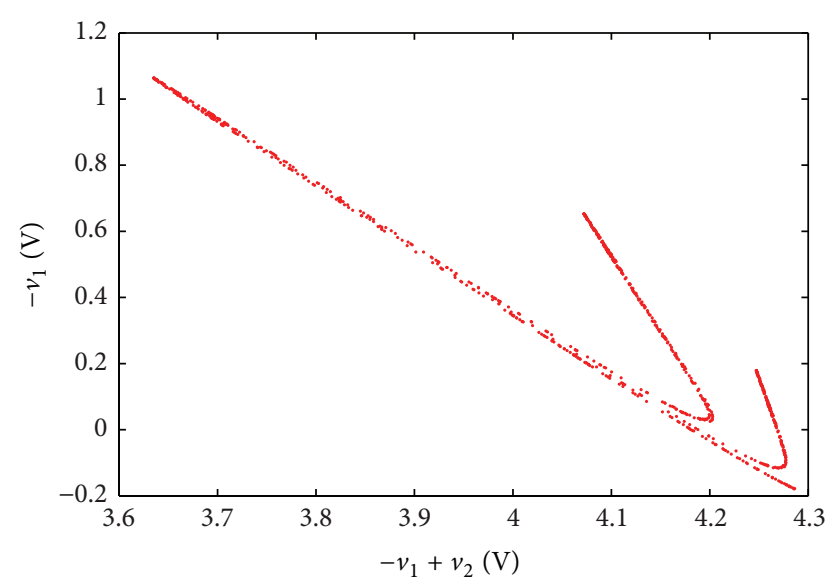

(a)
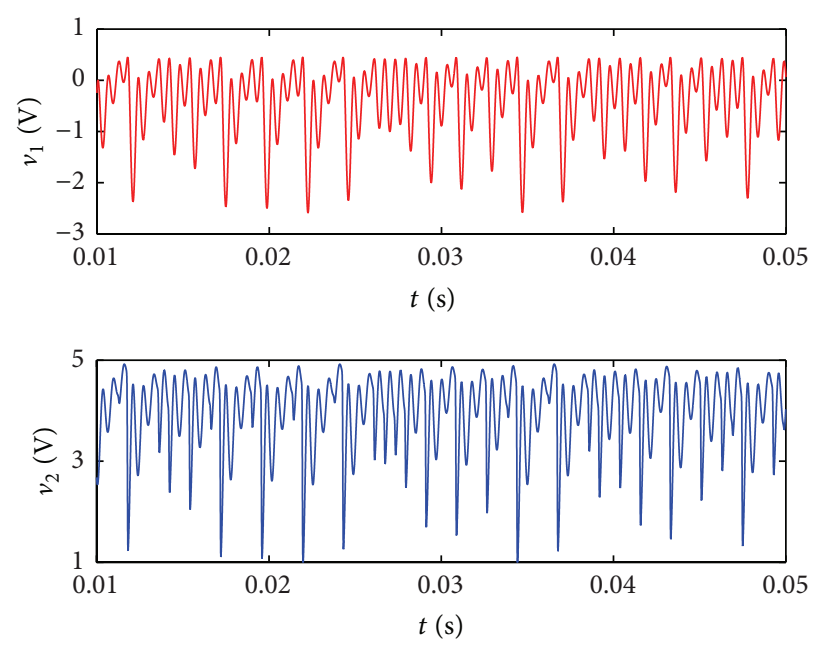

(c)

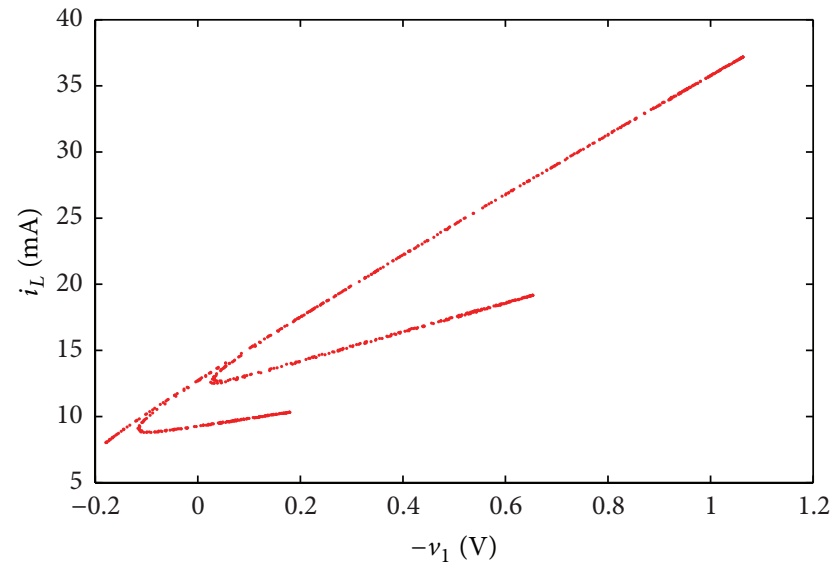

(b)
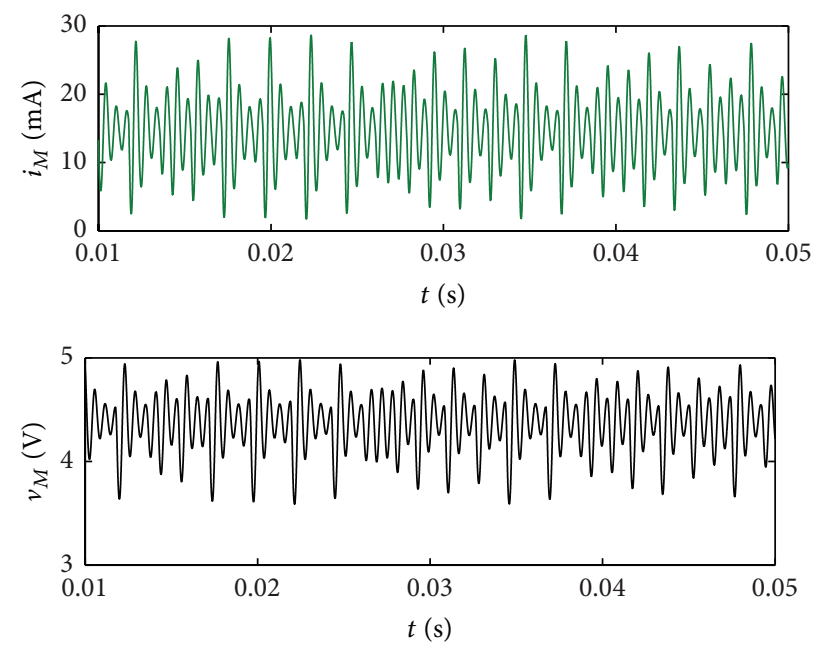

(d)

Figure 5: Poincaré mappings and time-domain waveforms: (a) Poincaré mapping in the $-v_{1}+v_{2}$ and $-v_{1}$ plane; (b) Poincaré mapping in the $-v_{1}$ and $i_{L}$ plane; (c) waveforms of $v_{1}(t)$ and $v_{2}(t)$; (d) waveforms of $i_{M}(t)$ and $v_{M}(t)$.

By numerical simulations, some typical chaotic and periodic orbits of the memristive Colpitts chaotic oscillator are obtained as shown in Figure 8. Figures 8(a) and 8(c) display two typical portrait phases in different chaotic regions, Figure 8(b) illustrates a typical limit cycle within periodic window, and Figure 8(d) demonstrates a normal orbit of period-2.

When the inner parameter $R_{0}$ of the generalized memristor is regarded as a bifurcation parameter, the bifurcation diagram of the state variable $v_{1}(t)$ and the corresponding Lyapunov exponent spectra of the memristive Colpitts oscillator are plotted in Figures 9(a) and 9(b), respectively. From Figure 9, it can be observed that, except for two periodic windows, chaotic behavior appearing in the memristive Colpitts oscillator has good robustness. Correspondingly, two typical portrait phases in the $v_{M}-i_{M}$ plane are shown in Figure 10, which demonstrate that the generalized memristor only exhibits positive right part of pinched hysteresis loops due to the applied positive stimuli.

\section{Conclusions}

In this paper, a novel memristive Colpitts chaotic oscillator is presented, which is derived from a fourth-order Colpitts chaotic oscillator coupling with a generalized memristor. The generalized memristor is equivalently realized by a diode bridge cascaded with a first-order parallel RC filter and its fingerprints of the pinched hysteresis loops are further elaborated. Theoretical analyses, numerical simulations, and experimental measurements of the memristive Colpitts chaotic oscillator are performed in order. The dynamical investigations indicate that the oscillator only has an unstable equilibrium point and displays complex nonlinear phenomena. The memristive Colpitts chaotic oscillator is simple and easy to implement physically, which can be used to generate chaotic signals for various engineering applications. 


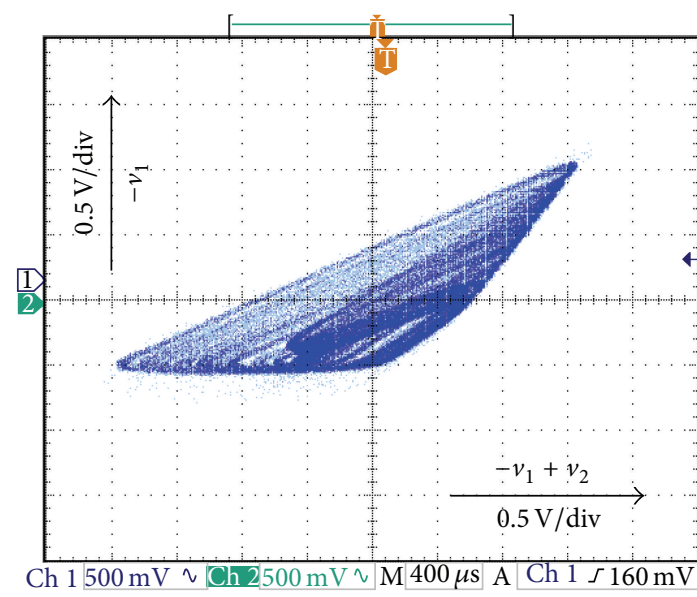

(a)

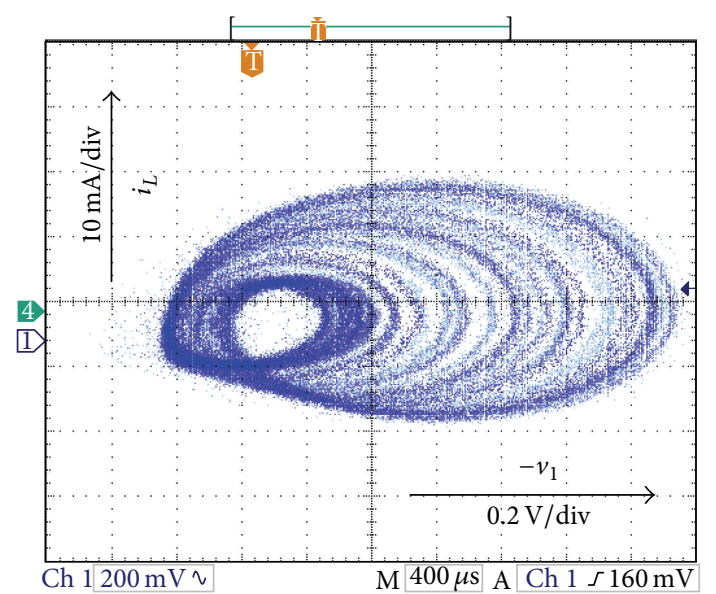

(c)

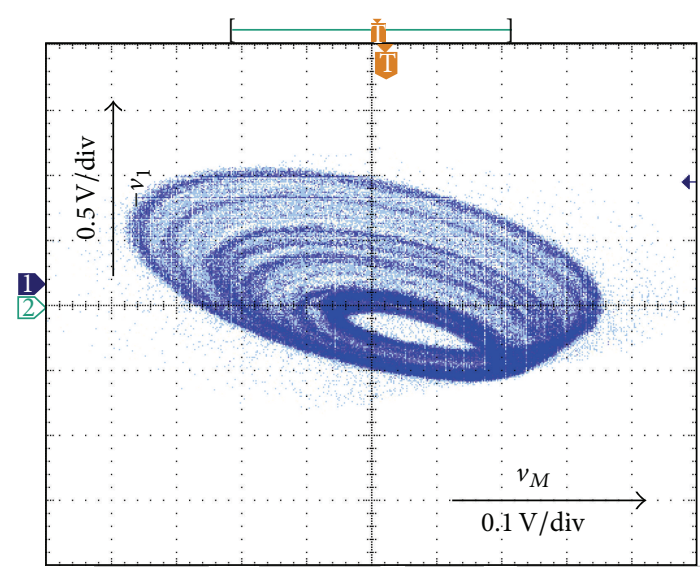

Ch $1100 \mathrm{mV} \sim$ Ch $2500 \mathrm{mV} \sim \mathrm{M} 400 \mu \mathrm{s}$ A Ch $1 \int 160 \mathrm{mV}$

(b)

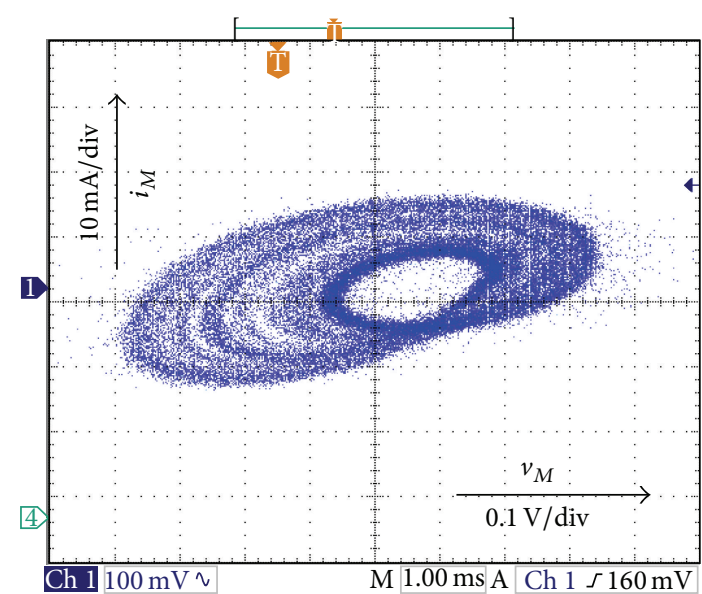

(d)

FIgURE 6: Phase portraits of the memristive Colpitts oscillator by experimental measurements: (a) $-v_{1}+v_{2}$ versus $-v_{1}$; (b) $v_{M}$ versus $-v_{1}$; (c) $-v_{1}$ versus $i_{L} ;(\mathrm{d}) v_{M}$ versus $i_{M}$.

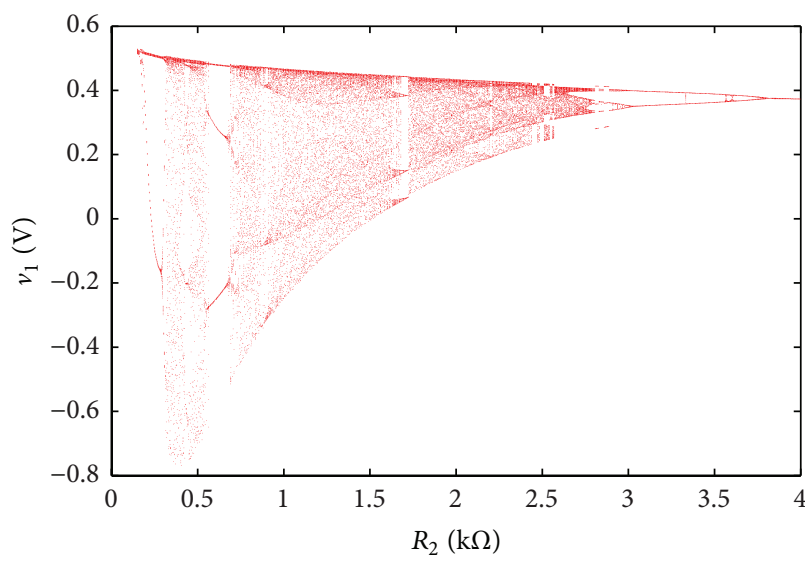

(a)

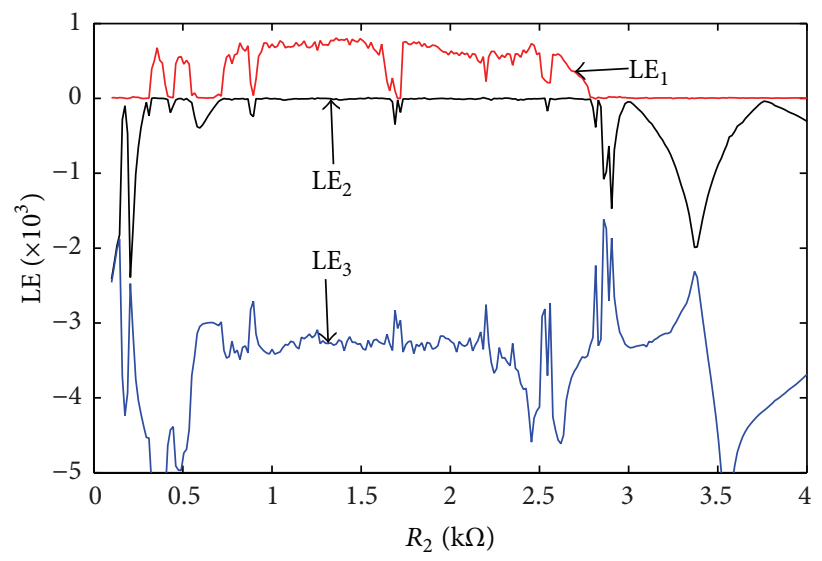

(b)

FIGURE 7: Dynamics with the resistance $R_{2}$ increasing: (a) bifurcation behavior of $v_{1}(t)$; (b) Lyapunov exponent spectra. 


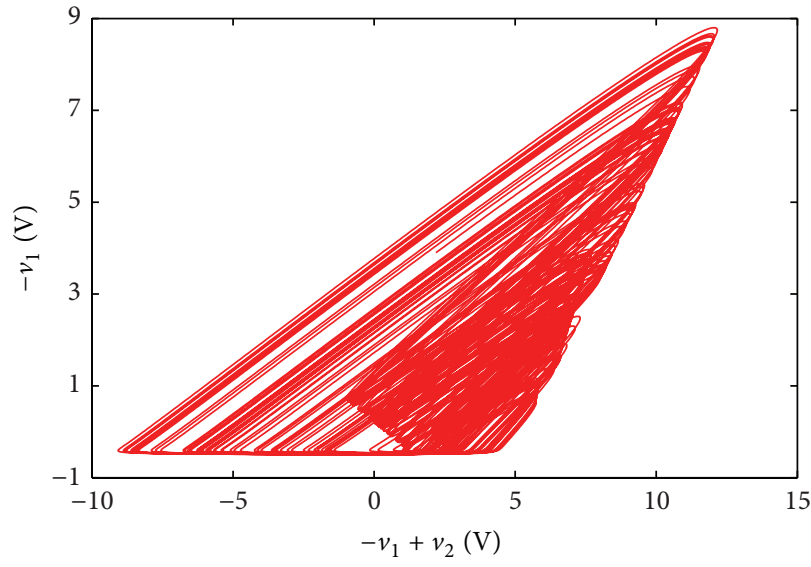

(a)

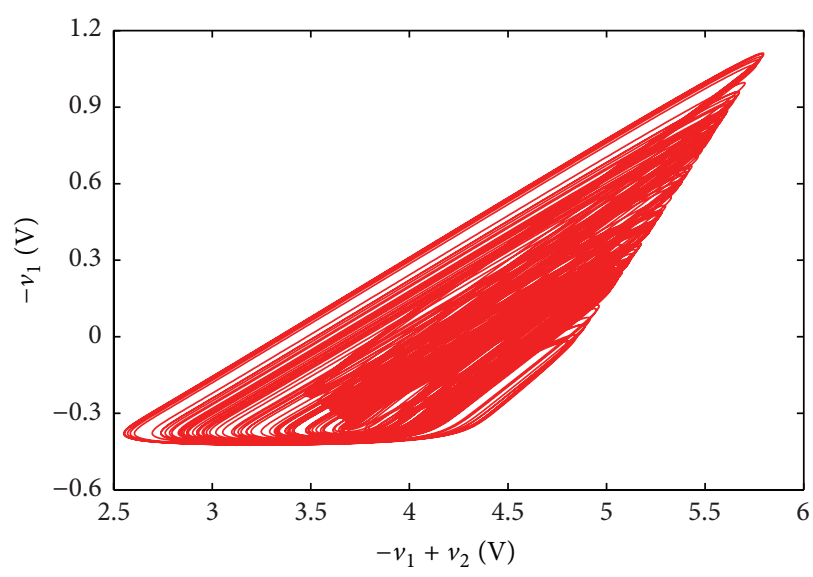

(c)

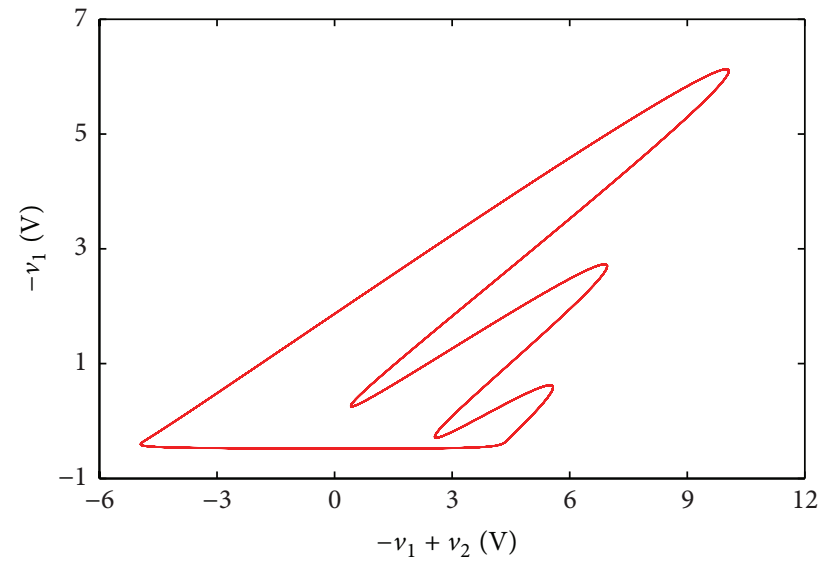

(b)

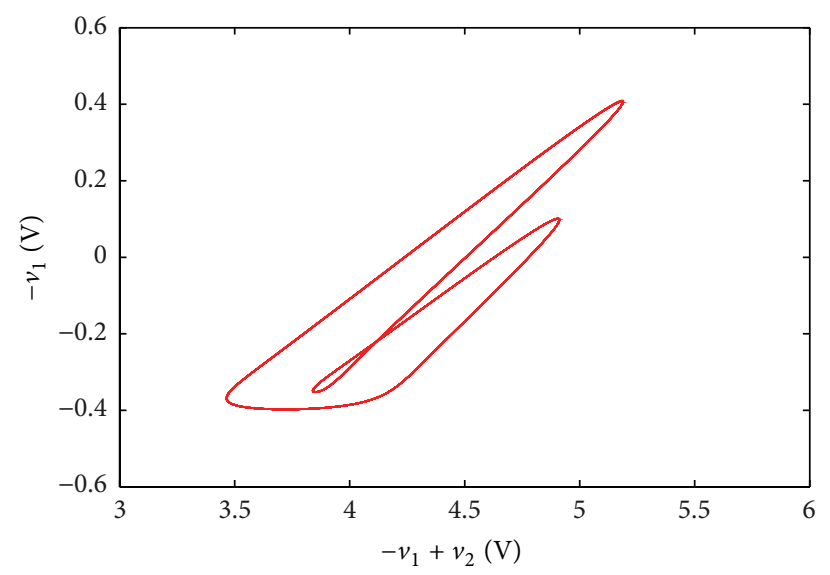

(d)

FIgure 8: Chaotic and periodic orbits in the $-v_{1}+v_{2}$ and $-v_{1}$ plane: (a) chaotic attractor $\left(R_{2}=0.5 \mathrm{k} \Omega\right.$ ); (b) period-3 limit cycle with $\left(R_{2}=0.6 \mathrm{k} \Omega\right)$; (c) chaotic attractor $\left(R_{2}=2.4 \mathrm{k} \Omega\right)$; (d) limit cycle with period-2 $\left(R_{2}=3.1 \mathrm{k} \Omega\right)$.

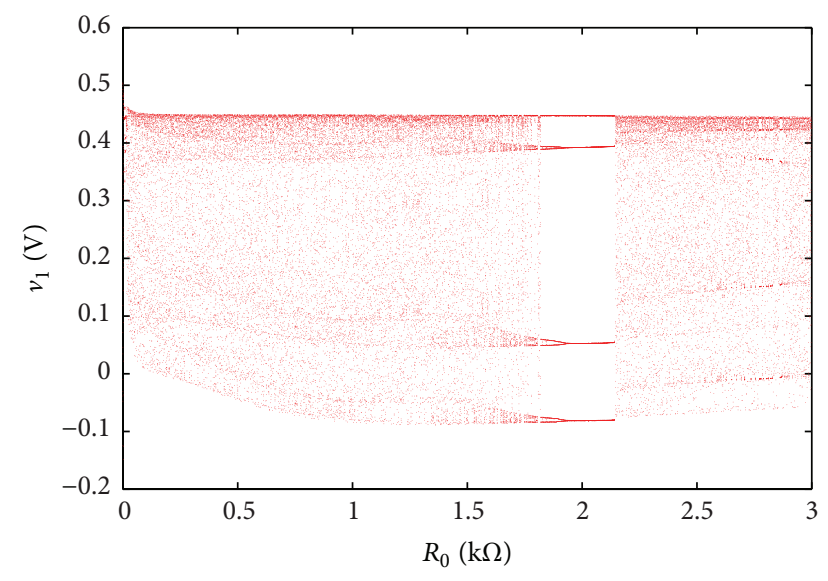

(a)

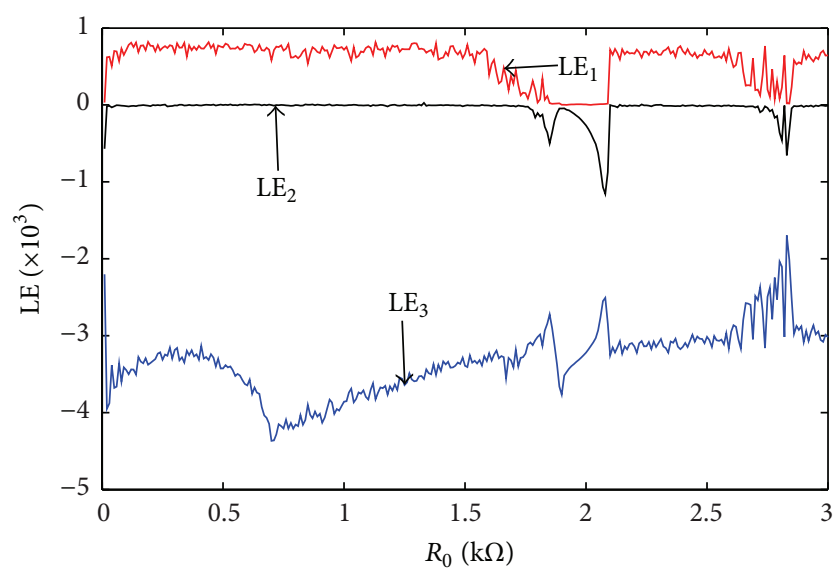

(b)

Figure 9: Dynamics with the resistance $R_{0}$ increasing: (a) bifurcation behavior of $v_{1}(t)$; (b) Lyapunov exponent spectra. 


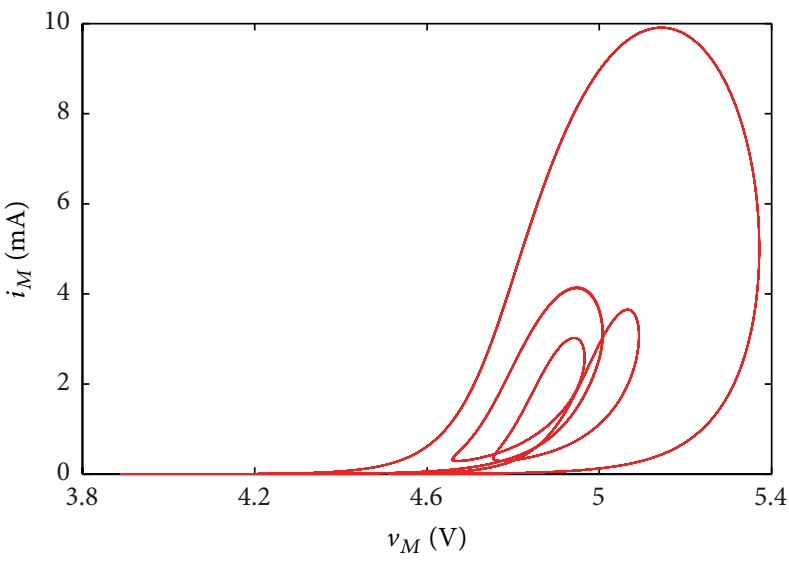

(a)

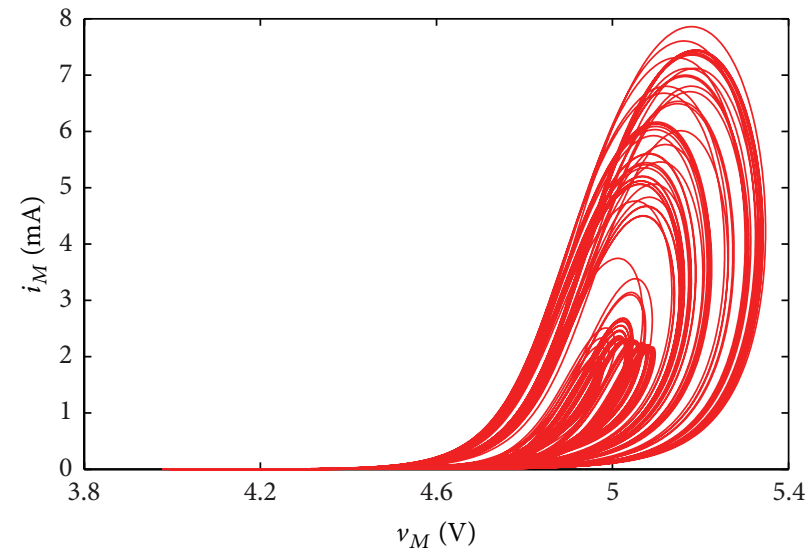

(b)

Figure 10: Periodic and chaotic orbits in the $v_{M}-i_{M}$ plane: (a) limit cycle with period-4 $\left(R_{2}=2 \mathrm{k} \Omega\right)$; (b) chaotic attractor $\left(R_{0}=3 \mathrm{k} \Omega\right)$.

\section{Conflict of Interests}

The authors declare that there is no conflict of interests regarding the publication of this paper.

\section{Acknowledgments}

This work is supported by the grants from the National Natural Science Foundations of China (Grant no. 51277017) and the Natural Science Foundations of Jiangsu Province (Grant no. BK20120583).

\section{References}

[1] D. B. Strukov, G. S. Snider, D. R. Stewart, and R. S. Williams, "The missing memristor found," Nature, vol. 453, pp. 80-83, 2008.

[2] L. O. Chua, "Memrister-the missing circuit element," IEEE Transactions on Circuit Theory, vol. 18, no. 5, pp. 507-519, 1971.

[3] B.-C. Bao, J.-P. Xu, and Z. Liu, "Initial state dependent dynamical behaviors in a memristor based chaotic circuit," Chinese Physics Letters, vol. 27, no. 7, Article ID 070504, 2010.

[4] Y. Li, X. Huang, and M. Guo, "The generation, analysis, and circuit implementation of a new memristor based chaotic system," Mathematical Problems in Engineering, vol. 2013, Article ID 398306, 8 pages, 2013.

[5] B. Muthuswamy, "Implementing memristor based chaotic circuits," International Journal of Bifurcation and Chaos, vol. 20, no. 5, pp. 1335-1350, 2010.

[6] G.-Y. Wang, J.-L. He, F. Yuan, and C.-J. Peng, "Dynamical behaviors of a $\mathrm{TiO}_{2}$ memristor oscillator," Chinese Physics Letters, vol. 30, no. 11, Article ID 110506, 2013.

[7] B.-C. Bao, J.-P. Xu, G.-H. Zhou, Z.-H. Ma, and L. Zou, "Chaotic memristive circuit: equivalent circuit realization and dynamical analysis," Chinese Physics B, vol. 20, no. 12, Article ID 120502, 2011.

[8] B. Bao, F. Hu, Z. Liu, and J. Xu, "Mapping equivalent approach to analysis and realization of memristor-based dynamical circuit," Chinese Physics B, vol. 23, no. 7, Article ID 070503, 2014.
[9] A. Buscarino, L. Fortuna, M. Frasca, and L. V. Gambuzza, "A chaotic circuit based on Hewlett-Packard memristor," Chaos, vol. 22, no. 2, Article ID 023136, 2012.

[10] B. C. Bao, Z. Liu, and J. P. Xu, "Steady periodic memristor oscillator with transient chaotic behaviours," Electronics Letters, vol. 46, no. 3, pp. 228-230, 2010.

[11] M. Chen, J. J. Yu, Q. Yu, C. D. Li, and B. C. Bao, "A memristive diode bridge-based canonical Chua's circuit," Entropy, vol. 16, no. 12, pp. 6464-6476, 2014.

[12] Z.-J. Li and Y.-C. Zeng, "A memristor oscillator based on a twinT network," Chinese Physics B, vol. 22, no. 4, Article ID 040502, 2013.

[13] B. Bao, Z. Ma, J. Xu, Z. Liu, and Q. Xu, "A simple memristor chaotic circuit with complex dynamics," International Journal of Bifurcation and Chaos, vol. 21, no. 9, pp. 2629-2645, 2011.

[14] G. M. Maggio, O. de Feo, and M. P. Kennedy, "Nonlinear analysis of the Colpitts oscillator and applications to design," IEEE Transactions on Circuits and Systems I: Fundamental Theory and Applications, vol. 46, no. 9, pp. 1118-1130, 1999.

[15] S.-M. Yu, "Fourth-order Colpitts chaotic oscillator," Acta Physica Sinica, vol. 57, no. 6, pp. 3374-3379, 2008 (Chinese).

[16] X. Y. Wang, A. L. Fitch, H. H. C. Iu, V. Sreeram, and W. G. Qi, "Implementation of an analogue model of a memristor based on a light-dependent resistor," Chinese Physics B, vol. 21, no. 10, Article ID 108501, 2012.

[17] X. Y. Wang, A. L. Fitch, H. H. C. Iu, and W. G. Qi, "Design of a memcapacitor emulator based on a memristor," Physics Letters A, vol. 376, no. 4, pp. 394-399, 2012.

[18] B. C. Bao, J. J. Yu, F. W. Hu, and Z. Liu, "Generalized memristor consisting of diode bridge with first order parallel RC filter," International Journal of Bifurcation and Chaos, vol. 24, no. 11, Article ID 1450143, 4 pages, 2014.

[19] F. Corinto and A. Ascoli, "Memristive diode bridge with LCR filter," Electronics Letters, vol. 48, no. 14, pp. 824-825, 2012.

[20] L. O. Chua, "The fourth element," Proceedings of the IEEE, vol. 100, no. 6, pp. 1920-1927, 2012.

[21] S. P. Adhikari, M. P. Sah, H. Kim, and L. O. Chua, "Three fingerprints of memristor," IEEE Transactions on Circuits and Systems I: Regular Papers, vol. 60, no. 11, pp. 3008-3021, 2013. 


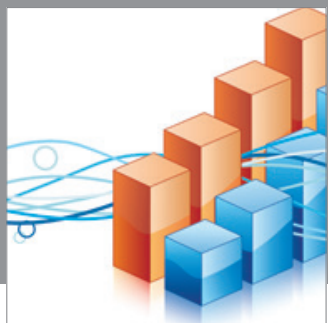

Advances in

Operations Research

mansans

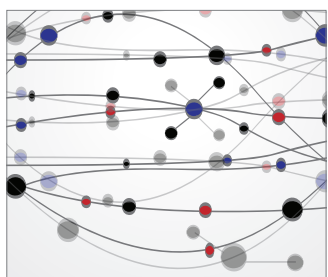

The Scientific World Journal
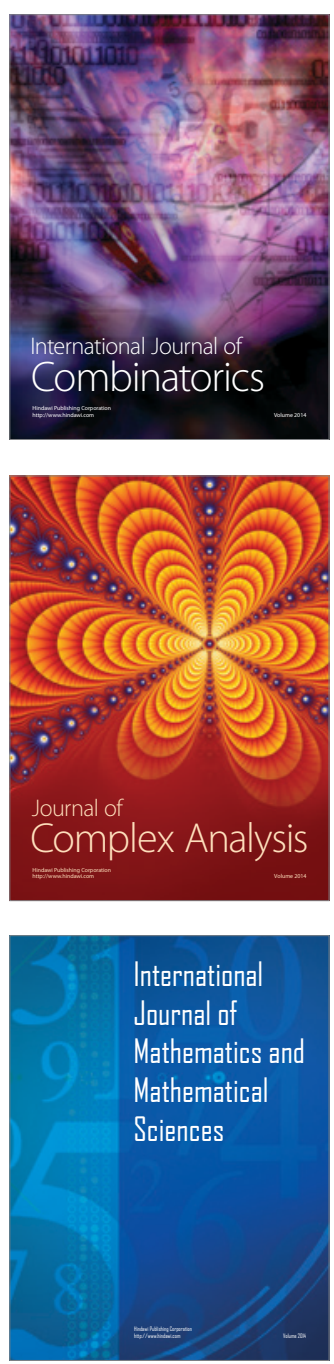
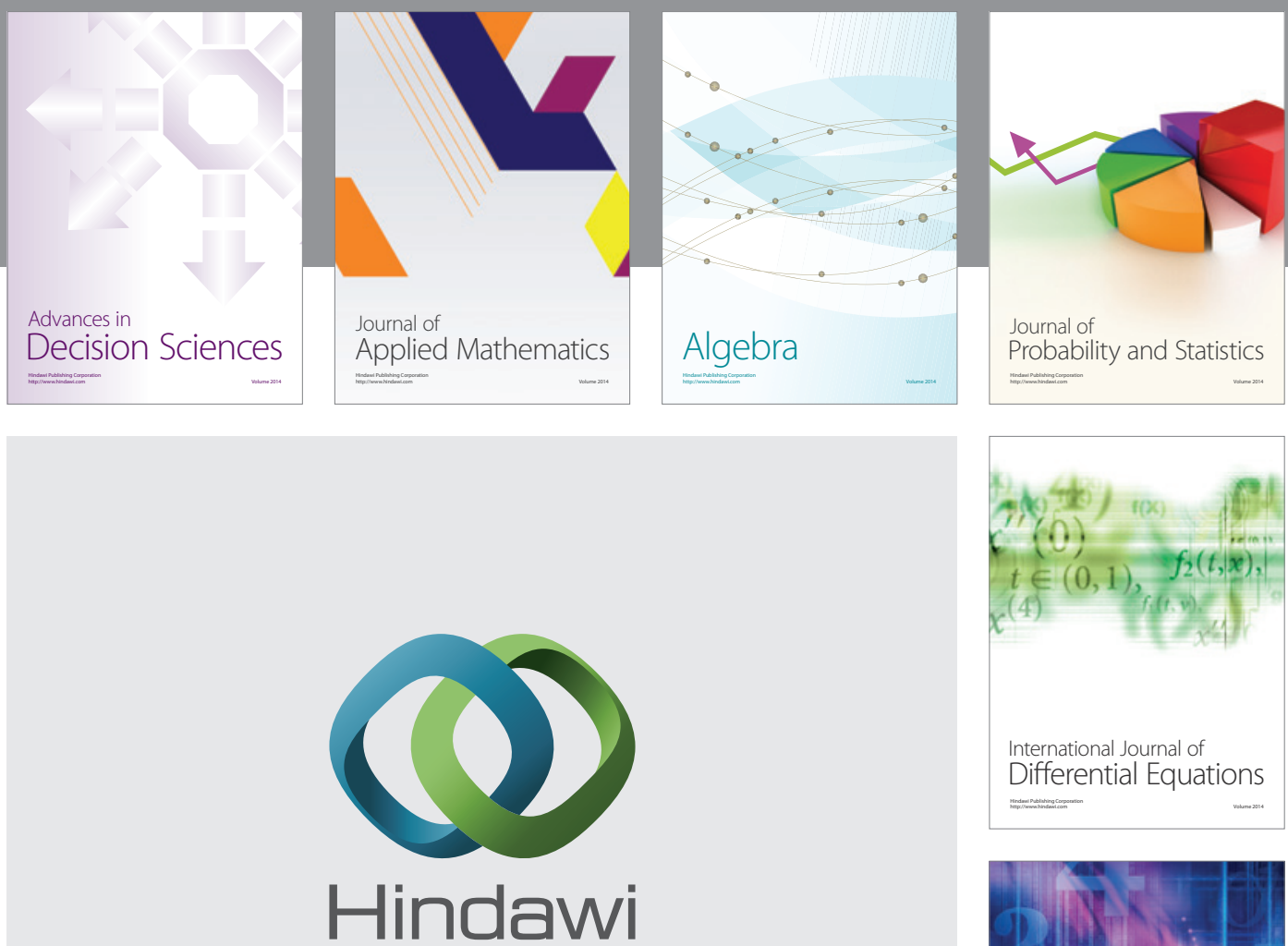

Submit your manuscripts at http://www.hindawi.com
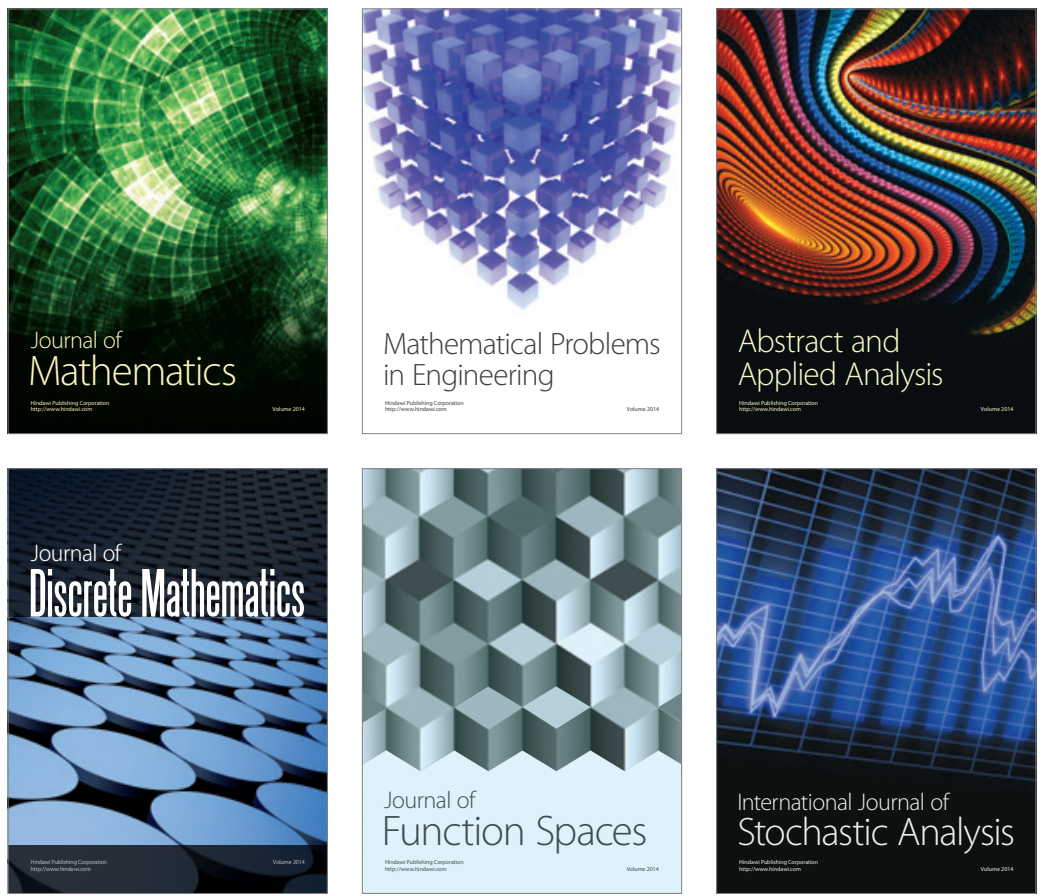

Journal of

Function Spaces

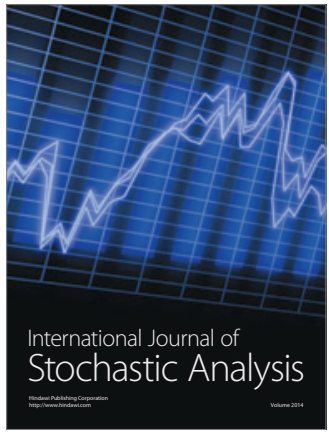

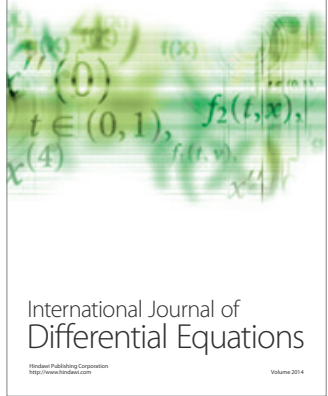
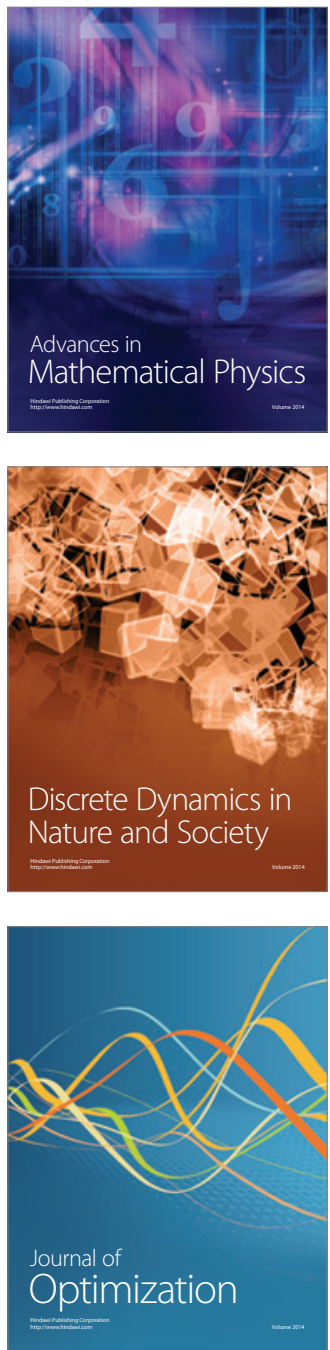